\title{
Primary care physician volume and quality of care for older adults with dementia: a retrospective cohort study
}

Natasha E. Lane ${ }^{1,2^{*}}$, Vicki Ling ${ }^{2}$, Richard H. Glazier ${ }^{2,3,4,5,6}$ and Thérèse A. Stukel $2,4,7$

\begin{abstract}
Background: Some jurisdictions restrict primary care physicians' daily patient volume to safeguard quality of care for complex patients. Our objective was to determine whether people with dementia receive lower-quality care if their primary care physician sees many patients daily.

Methods: Population-based retrospective cohort study using health administrative data from 100,256 communityliving adults with dementia aged 66 years or older, and the 8,368 primary care physicians who cared for them in Ontario, Canada. Multivariable Poisson GEE regression models tested whether physicians' daily patient volume was associated with the adjusted likelihood of people with dementia receiving vaccinations, prescriptions for cholinesterase inhibitors, benzodiazepines, and antipsychotics from their primary care physician.
\end{abstract}

Results: People with dementia whose primary care physicians saw $\geq 30$ patients daily were $32 \%$ (95\% Cl: 23\% to 41\%, $p<0.0001)$ and $25 \%$ (95\% Cl: $17 \%$ to 33\%, $p<0.0001)$ more likely to be prescribed benzodiazepines and antipsychotic medications, respectively, than patients of primary care physicians who saw $<20$ patients daily. Patients were 3\% $195 \%$ Cl: $0.4 \%$ to $6 \%, p=0.02)$ less likely to receive influenza vaccination and $8 \%(95 \% \mathrm{Cl}: 4 \%$ to $13 \%, p=0.0001)$ more likely to be prescribed cholinesterase inhibitors if their primary care physician saw $\geq 30$ versus $<20$ patients daily.

Conclusions: People with dementia were more likely to receive both potentially harmful and potentially beneficial medications, and slightly less likely to be vaccinated by high-volume primary care physicians.

Keywords: Dementia, Patient volume, Quality indicators, Primary health care

\section{Background}

In 2015, 46 million people in the world had dementia; this number is expected to double every 20 years as the global population ages, reaching 131.5 million by 2050 [1]. Up to $70 \%$ of older adults with dementia live in private community dwellings [2] and the majority of their care is provided by primary care physicians (PCPs) $[3,4]$. Caring for older adults with dementia is complex; most have numerous medical comorbidities and are more likely to

\footnotetext{
*Correspondence: natasha.lane@alumni.ubc.ca

1 Department of Medicine, University of British Columbia, British

Columbia, 2775 Laurel Street, 10th Floor, Vancouver V5Z 1M9, Canada

Full list of author information is available at the end of the article
}

experience adverse side effects to commonly prescribed medicines [5]. As the prevalence of dementia increases globally, PCPs and their governing bodies urgently need to understand how to structure primary care to provide older adults with dementia the care they require. Daily patient volume is a policy-sensitive aspect of primary care delivery [6]; some jurisdictions cap the number of patients PCPs may bill for seeing each day [7] and other regions are considering similar restrictions [8]. The effect of PCPs' daily patient volume on quality of dementia care is unknown.

Quality primary care for people with dementia arises from judicious provision of helpful interventions and withholding or withdrawing potentially harmful ones.

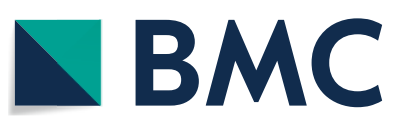

(c) The Author(s) 2021. Open Access This article is licensed under a Creative Commons Attribution 4.0 International License, which permits use, sharing, adaptation, distribution and reproduction in any medium or format, as long as you give appropriate credit to the original author(s) and the source, provide a link to the Creative Commons licence, and indicate if changes were made. The images or other third party material in this article are included in the article's Creative Commons licence, unless indicated otherwise in a credit line to the material. If material is not included in the article's Creative Commons licence and your intended use is not permitted by statutory regulation or exceeds the permitted use, you will need to obtain permission directly from the copyright holder. To view a copy of this licence, visit http://creativecommons.org/licenses/by/4.0/. The Creative Commons Public Domain Dedication waiver (http://creativeco $\mathrm{mmons}$.org/publicdomain/zero/1.0/) applies to the data made available in this article, unless otherwise stated in a credit line to the data. 
PCPs with high daily patient volumes are known to spend significantly less time (8.8 versus 12.5 min per visit) with each patient, [9] bill fewer service codes that require long visits, [10] and rely more heavily on non-physician staff to refill prescriptions [11] than low-volume PCPs. In keeping with Burgess' cognitive burden framework, we hypothesized that these time pressures and organizational routines associated with increased daily patient volume would compromise PCP's ability to partake in the "controlled processing" necessary to optimize care in complex patients with dementia [12]. This deleterious effect of high daily patient volume on quality of primary care has been demonstrated in the management of other conditions: high-volume PCPs inappropriately prescribe antibiotics to treat viral infections at a higher rate than their low-volume counterparts; $[13,14]$ they also provide lower-quality preventive care and medication management to people with asthma, angina, and diabetes than physicians who see fewer patients per day $[6,15,16]$. Even if high-volume PCPs were able to achieve controlled processing with reduced visit length, we posit that they would have less time to have the sometimes-difficult conversations around starting or stopping medications that target the neuropsychiatric symptoms of dementia [17].

Our main objective was to determine the association between PCP's daily patient volume and provision of quality primary care to their patients with dementia. We examined annual vaccination against influenza and cholinesterase inhibitor prescriptions as indicators of good quality care, given their favourable risk-benefit ratio in most older adults with dementia $[18,19]$. Benzodiazepine and antipsychotic prescriptions were selected as indicators of lower quality primary care because their adverse side-effect profile supports extremely stringent use in this population, [20] despite their indication in select patients with dementia [21]. Our secondary objective was to assess whether patients' annual visit frequency, continuity of care, and long-term relationships with their PCP benefited patients of high-volume PCPs more than those of low-volume PCPs. Our hypothesis was that these features of the doctor-patient relationship might reduce cognitive burden in high-volume physicians and afford the controlled processing and communication time necessary to optimize complex patient care during brief office visits.

\section{Methods.}

\section{Study Design and Setting}

We conducted a population-based retrospective cohort study of 100,256 older adults with dementia and the 8,368 PCPs who cared for them. This study was set in Ontario, Canada, where medically necessary physician and hospital care is publicly insured for all residents, as are medications for adults 65 years of age and older. This manuscript is reported in accordance with the STROBE and RECORD statements for the reporting of observational studies conducted with health administrative data [22, 23].

\section{Data Sources}

We created our cohort using datasets that were linked using unique encoded identifiers and analyzed at ICES. ICES is an independent, non-profit research institute whose legal status under Ontario's health information privacy law allows it to collect and analyze health care and demographic data, without consent, for health system evaluation and improvement. The following Ontario health administrative databases were included: Registered Persons Database, Ontario Health Insurance Plan Database (OHIP), [24] Discharge Abstract Database, [25] Ontario Drug Benefit Database (ODB), [26] National Ambulatory Care Reporting System, [27] the rurality index of Ontario, [28] ICES Physician Database, [29] Client Agency Program Enrollment, and Ontario Marginalization Index, [30] and the Immigration, Refugees and Citizenship Canada Permanent Resident (IRCC-PR) database [31]. A description of these databases and their contents is available in Additional file 1.

\section{Study Population}

We enrolled people aged 66 years or older who had dementia as of April 1, 2016, the index study date, and received care from a comprehensive PCP [32]. Dementia diagnosis was determined using a validated algorithm with $79.3 \%$ sensitivity and $99.1 \%$ specificity [33]. Patients were excluded if they were not Ontario residents or did not have access to provincial health insurance in the previous three years, or were residents of long-term care homes (Fig. 1). Our observation period was one year, with a maximum follow-up date of March 31, 2017. Baseline characteristics and doctor-patient relationship variables were determined in the two years prior to index date, unless specified otherwise. PCPs were included if they were the designated $\mathrm{PCP}$ for at least one study participant [34] and met the criteria for a comprehensive PCP [32] in at least one year from April 1, 2013 to March 31, 2016.

\section{Measures}

\section{Outcomes.}

We defined receipt of influenza vaccine using validated OHIP codes with $74.3 \%$ sensitivity and $73.8 \%$ specificity among people with dementia [35]. Filled prescriptions from patients' PCPs for cholinesterase inhibitors, antipsychotic medications and benzodiazepines in the year following index date were identified from the ODB. See Additional file 2 for a complete list of diagnostic codes and drug names used to classify these study variables. 


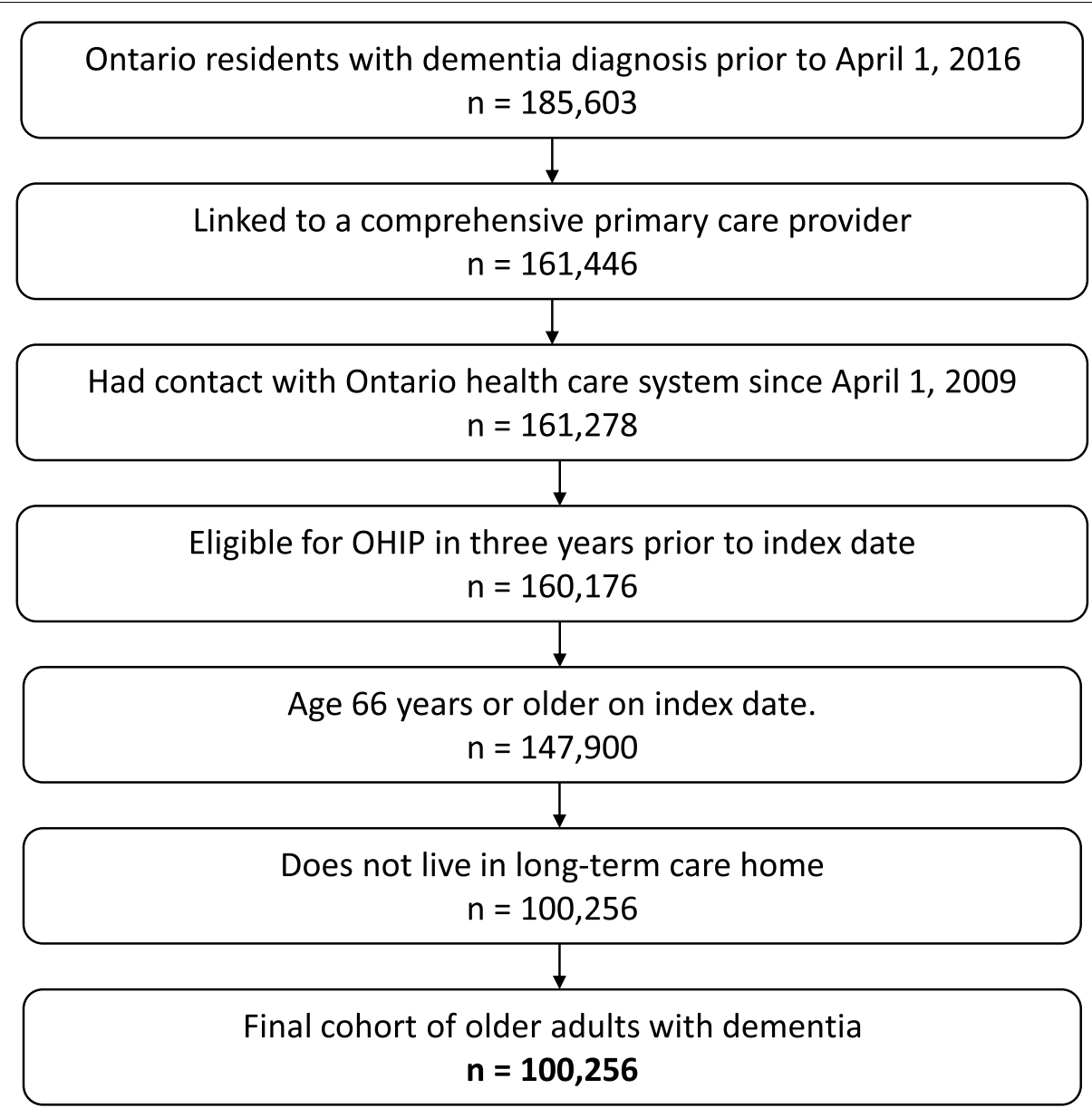

Fig. 1 Study Flow Diagram

\section{Exposure.}

Patients were assigned to the PCP to whom they were rostered or virtually rostered [34]. The exposure of interest was the mean number of patients seen per day by the patient's PCP (henceforth "daily patient volume"). Daily patient volume was calculated as the total number of unique office visits to a PCP (by all patients, not just those with dementia) between April 1, 2013 and March 31, 2016, divided by the total number of dates on which those visits took place [6]. We chose $<20,20-24$, 25-29 and $\geq 30$ patients per day as a priori volume cutoffs, so that "high-volume" PCPs were those who saw 30 or more patients per day and "low-volume" PCPs saw fewer than 20 patients per day. We chose these cutpoints to bracket the commonly quoted estimate that primary care physicians can reasonably care for about 25 patients per day $[8,36]$.

\section{Doctor-Patient Relationship Variables}

We also assessed whether the effects of doctor-patient relationship on outcomes was the same for patients of high- versus low-volume PCPs. These were: (a) high visit frequency, defined as seven or more visits per year to the PCP (the $75^{\text {th }}$ percentile in our sample) in the two years prior to the index date [37]; (b) high continuity of care, defined as patients seeing their PCP for $75 \%$ or more of all physician visits in the two years prior to index [38]; and (c) long doctor-patient relationship, defined as patients being attached to their PCP for five or more years [39]. Main effects of doctor-patient relationship variables were examined to facilitate interpretation of their interactions with daily patient volume.

\section{Confounders}

Patient characteristics adjusted for in multivariable models include age group-sex interactions, years since dementia diagnosis, CIHI Population Grouper comorbidity index [40] urban/rural location of residence, neighborhood deprivation quintile, and immigration to Canada in the past 10 years [31]. We also adjusted for outcome-specific variables such as past-year hospitalizations for chronic obstructive pulmonary disease (COPD) 
in influenza vaccine models. PCP variables adjusted for were age, sex, years since graduation, Canadian medical training, and urban/rural location of practice. Patient characteristics in the causal pathway (i.e., past-year medication and health care use, specialist visits) and PCP panel size and payment model were included in descriptive analyses but not regression models. Data on type of dementia (i.e., Alzheimer's, vascular, etc.), were only available for persons who had been hospitalized in the years leading up to the index data, therefore these data were described but omitted from analyses.

\section{Statistical Analysis}

The unit of analysis was the individual patient with dementia and the exposure was their PCP's daily patient volume. All analyses were pre-specified based on our a priori hypotheses. We reported baseline characteristics according to daily patient volume groups. We estimated the confounder-adjusted relative rate of influenza vaccination, cholinesterase inhibitor prescription, benzodiazepine prescription and antipsychotic prescription separately using Poisson GEE models that accounted for clustering of patients within PCPs. Patients who died during follow-up were censored on the date of death. We also assessed main effects of doctor-patient relationship variables and their interactions with daily patient volume.

Secondary analyses examined the proportion of patients in each PCP volume group who changed physicians during the study period, as well as the effect of daily patient volume on medications prescribed by all physicians (not just PCPs). Multivariable analyses were completed using proc gee [41] in SAS version 9.04.

\section{Results}

Our cohort consisted of 100,256 community-living older adults with dementia (Fig. 1), among whom 58,993 (59\%) were female and $40,928(41 \%)$ were 85 years or older. People were cared for by 8,368 comprehensive PCPs (45\% female) who had a median panel size of 1,281 (IQR: $856-1,790)$ patients. The median daily patient volume was 23 (IQR: 18-31) patients per day (Table A, Additional file 3). Patients of high-volume PCPs ( $\geq 30$ patients per day) were more likely to come from deprived neighborhoods and live in urban settings than patients whose PCPs saw fewer than 20 patients daily (Table 1). The number of unique medications taken at index date and past-year benzodiazepine and antipsychotic use were higher among patients of high-volume PCPs. There were no significant differences in proportion of people who had been treated by psychiatrists, geriatricians or neurologists in the year prior to index date, nor were there differences in past-year rates of hospitalizations or emergency department visits. People with dementia who had high-volume PCPs had an average of 13 visits per year to their PCP, as compared with eight visits/year among patients of low-volume PCPs. During the study year, 6,950 (6.9\%) of people changed PCPs, with no differences in rate of switching across daily patient volume groups (Table B, Additional file 3).

High-volume PCPs were significantly different from PCPs who saw fewer than 20 patients daily: their median panel size was more than twice as large $(2,002$ vs 977 patients) and they were more likely to be older, male, Canadian medical graduates, and practice in urban areas (Table 2). More than three quarters $(75.3 \%)$ of high-volume PCPs were remunerated on a primarily fee-for-service basis, compared to only $25.6 \%$ of low-volume PCPs.

In our primary analysis (Table 3), older adults with dementia were 1.32 (95\% CI: 1.23 to 1.41 ) and 1.25 (95\% CI: 1.17 to 1.33 ) times more likely to receive benzodiazepine and antipsychotic prescriptions from high-volume PCPs than low-volume PCPs (Table 3; full model in Table $\mathrm{D}$, Additional file 3). They were also more likely (aRR $1.08,95 \% \mathrm{CI}: 1.04$ to 1.13 ) to receive cholinesterase inhibitor prescriptions and slightly less likely (aRR 0.97, 95\% CI: 0.94 to 0.996 ) to be vaccinated against influenza than people with dementia cared for by low-volume PCPs. Patients received most prescriptions for cholinesterase inhibitors, benzodiazepines and antipsychotics from their PCP (Table C, Additional file 3) and the magnitude of the volume-outcome relationships was diminished significantly when we included prescriptions from all physicians (Table E, Additional file 3).

High visit frequency and high continuity of care were associated with an increased likelihood of influenza vaccination and higher levels of prescribing of all medications, adjusting for physician volume (Table 4). Patients who had known their doctor for at least five years were more likely to receive influenza vaccination and cholinesterase inhibitor prescriptions, but less likely (aRR $0.89,95 \% \mathrm{CI}: 0.85$ to 0.94 ) to be prescribed antipsychotic medications, adjusting for daily patient volume and confounders. There were no significant interactions between the daily patient volume and the doctor-patient relationship variables with respect to prescribing of benzodiazepines or antipsychotics. There was a significant interaction between physician volume and both patient visit frequency and continuity of care, serving to increase the relative provision of cholinesterase inhibitors and influenza vaccinations by high compared to low volume physicians by about $8-15 \%$ (Table F, Additional file 3).

\section{Discussion}

In keeping with our cognitive burden hypothesis, we found that - controlling for morbidity and time since dementia diagnosis - community-dwelling people with 
Table 1 Baseline Characteristics of 100,256 Community-living Ontarians with Dementia, by PCP's daily patient volume

\begin{tabular}{|c|c|c|c|c|c|}
\hline \multirow[b]{3}{*}{ Patient Characteristics } & \multicolumn{4}{|c|}{ Average Number of Patients Seen Daily by Patient's Primary Care Physician } & \multirow{3}{*}{$\begin{array}{l}\text { Standardizesso } \\
\text { Difference } \\
(<20 \text { vs } \geq 30)\end{array}$} \\
\hline & $<20$ & $20-24$ & $25-29$ & $\geq 30$ & \\
\hline & $\begin{array}{l}N=36,150 \\
(\%)\end{array}$ & $\begin{array}{l}N=21,362 \\
(\%)\end{array}$ & $\begin{array}{l}N=15,224 \\
(\%)\end{array}$ & $\begin{array}{l}N=27,520 \\
(\%)\end{array}$ & \\
\hline \multicolumn{6}{|l|}{ Age group } \\
\hline $66-69$ & $2,078(5.7 \%)$ & $1,160(5.4 \%)$ & $822(5.4 \%)$ & $1,659(6.0 \%)$ & 0.01 \\
\hline $70-74$ & $4,034(11.2 \%)$ & $2,270(10.6 \%)$ & 1,704 (11.2\%) & $3,310(12.0 \%)$ & 0.03 \\
\hline $75-79$ & $6,373(17.6 \%)$ & $3,800(17.8 \%)$ & $2,607(17.1 \%)$ & $4,960(18.0 \%)$ & 0.01 \\
\hline $80-84$ & $8,768(24.3 \%)$ & $5,310(24.9 \%)$ & $3,691(24.2 \%)$ & $6,782(24.6 \%)$ & 0.01 \\
\hline $85+$ & $14,897(41.2 \%)$ & $8,822(41.3 \%)$ & $6,400(42.0 \%)$ & $10,809(39.3 \%)$ & 0.04 \\
\hline \multicolumn{6}{|l|}{ Sex } \\
\hline Female & $21,794(60.3 \%)$ & $12,598(59.0 \%)$ & $8,779(57.7 \%)$ & $15,822(57.5 \%)$ & 0.06 \\
\hline Male & $14,356(39.7 \%)$ & $8,764(41.0 \%)$ & $6,445(42.3 \%)$ & $11,698(42.5 \%)$ & 0.06 \\
\hline \multicolumn{6}{|l|}{ Neighborhood Deprivation Index } \\
\hline 1-least deprived & $6,422(17.8 \%)$ & $3,554(16.6 \%)$ & $2,480(16.3 \%)$ & $3,396(12.3 \%)$ & 0.15 \\
\hline 2 & $7,104(19.7 \%)$ & $3,994(18.7 \%)$ & $2,822(18.5 \%)$ & 4,332 (15.7\%) & 0.10 \\
\hline 3 & 7,065 (19.5\%) & $4,186(19.6 \%)$ & $2,868(18.8 \%)$ & $4,946(18.0 \%)$ & 0.04 \\
\hline 4 & $7,296(20.2 \%)$ & $4,295(20.1 \%)$ & $3,154(20.7 \%)$ & $6,442(23.4 \%)$ & 0.08 \\
\hline 5-most deprived & $7,431(20.6 \%)$ & $4,886(22.9 \%)$ & $3,538(23.2 \%)$ & $7,618(27.7 \%)$ & 0.17 \\
\hline Missing data & $832(2.3 \%)$ & $447(2.1 \%)$ & $362(2.4 \%)$ & $786(2.9 \%)$ & 0.03 \\
\hline Immigrated to Ontario in past 10 years & $561(1.6 \%)$ & $346(1.6 \%)$ & $278(1.8 \%)$ & $753(2.7 \%)$ & 0.08 \\
\hline \multicolumn{6}{|l|}{ Location of dwelling } \\
\hline Urban & $25,141(69.5 \%)$ & $15,604(73.0 \%)$ & $11,533(75.8 \%)$ & $22,482(81.7 \%)$ & 0.29 \\
\hline Non-urban & $7,328(20.3 \%)$ & $4,476(21.0 \%)$ & $3,034(19.9 \%)$ & $4,139(15.0 \%)$ & 0.14 \\
\hline Rural & $3,478(9.6 \%)$ & $1,227(5.7 \%)$ & $615(4.0 \%)$ & $818(3.0 \%)$ & 0.28 \\
\hline Missing data & $203(0.6 \%)$ & $55(0.3 \%)$ & $42(0.3 \%)$ & $81(0.3 \%)$ & 0.04 \\
\hline \multicolumn{6}{|c|}{ CIHI Comorbidity Group Index (2-year look-back) } \\
\hline No health condition & $328(0.9 \%)$ & $189(0.9 \%)$ & $142(0.9 \%)$ & $187(0.7 \%)$ & 0.03 \\
\hline $\begin{array}{l}\text { Major and } \\
\text { palliative }\end{array}$ & $27,750(76.8 \%)$ & $16,028(75.0 \%)$ & $11,345(74.5 \%)$ & $19,888(72.3 \%)$ & 0.10 \\
\hline Moderate & $5,193(14.4 \%)$ & $3,356(15.7 \%)$ & 2,387 (15.7\%) & $4,741(17.2 \%)$ & 0.08 \\
\hline Minor & $2,570(7.1 \%)$ & $1,564(7.3 \%)$ & $1,130(7.4 \%)$ & $2,226(8.1 \%)$ & 0.04 \\
\hline Non-user & $309(0.9 \%)$ & $225(1.1 \%)$ & $220(1.4 \%)$ & $478(1.7 \%)$ & 0.08 \\
\hline \multicolumn{6}{|l|}{ Type of Dementia } \\
\hline Alzheimer's & $2,864(7.9 \%)$ & $1,665(7.8 \%)$ & $1,169(7.7 \%)$ & $2,100(7.6 \%)$ & 0.01 \\
\hline Vascular & $786(2.2 \%)$ & $474(2.2 \%)$ & $309(2.0 \%)$ & $580(2.1 \%)$ & 0.00 \\
\hline Other & $9,119(25.2 \%)$ & $5,484(25.7 \%)$ & $3,945(25.9 \%)$ & $6,896(25.1 \%)$ & 0.00 \\
\hline Missing data & $23,381(64.7 \%)$ & $13,739(64.3 \%)$ & $9,801(64.4 \%)$ & $17,944(65.2 \%)$ & 0.01 \\
\hline \multicolumn{6}{|l|}{ Years since dementia diagnosis } \\
\hline $0-1$ & $14,456(40.0 \%)$ & $8,190(38.3 \%)$ & $5,851(38.4 \%)$ & $10,158(36.9 \%)$ & 0.06 \\
\hline $2-9$ & 19,415 (53.7\%) & $11,744(55.0 \%)$ & $8,385(55.1 \%)$ & $15,316(55.7 \%)$ & 0.04 \\
\hline$\geq 10$ & $2,279(6.3 \%)$ & $1,428(6.7 \%)$ & $988(6.5 \%)$ & $2,046(7.4 \%)$ & 0.04 \\
\hline \multicolumn{6}{|l|}{ \# unique medications in use on index date } \\
\hline $0-5$ & $7,015(19.4 \%)$ & $4,011(18.8 \%)$ & $2,679(17.6 \%)$ & $4,534(16.5 \%)$ & 0.08 \\
\hline $6-9$ & $8,468(23.4 \%)$ & $4,851(22.7 \%)$ & $3,311(21.7 \%)$ & $5,538(20.1 \%)$ & 0.08 \\
\hline $10-19$ & $15,372(42.5 \%)$ & $9,254(43.3 \%)$ & $6,542(43.0 \%)$ & $12,128(44.1 \%)$ & 0.03 \\
\hline $20+$ & $5,295(14.6 \%)$ & $3,246(15.2 \%)$ & $2,692(17.7 \%)$ & $5,320(19.3 \%)$ & 0.12 \\
\hline
\end{tabular}




\begin{tabular}{|c|c|c|c|c|c|}
\hline \multirow[b]{3}{*}{ Patient Characteristics } & \multicolumn{4}{|c|}{ Average Number of Patients Seen Daily by Patient's Primary Care Physician } & \multirow{3}{*}{$\begin{array}{l}\text { Standardizessd } \\
\text { Difference } \\
(<20 \text { vs } \geq 30)\end{array}$} \\
\hline & $<20$ & $20-24$ & $25-29$ & $\geq 30$ & \\
\hline & $\begin{array}{l}N=36,150 \\
(\%)\end{array}$ & $\begin{array}{l}N=21,362 \\
(\%)\end{array}$ & $\begin{array}{l}N=15,224 \\
(\%)\end{array}$ & $\begin{array}{l}N=27,520 \\
(\%)\end{array}$ & \\
\hline \multicolumn{6}{|l|}{ Medications taken in year prior to index date } \\
\hline $\begin{array}{l}\text { Cholinesterase } \\
\text { inhibitor }\end{array}$ & $14,942(41.3 \%)$ & $9,265(43.4 \%)$ & $6,729(44.2 \%)$ & $12,112(44.0 \%)$ & 0.05 \\
\hline Benzodiazepine & $4,864(13.5 \%)$ & $3,234(15.1 \%)$ & $2,451(16.1 \%)$ & $4,989(18.1 \%)$ & 0.13 \\
\hline Antipsychotic & $5,273(14.6 \%)$ & $3,319(15.5 \%)$ & $2,489(16.3 \%)$ & $5,114(18.6 \%)$ & 0.11 \\
\hline \multicolumn{6}{|l|}{ Patient Rostering to PCP } \\
\hline Not rostered & $1,728(4.8 \%)$ & $750(3.5 \%)$ & $759(5.0 \%)$ & $2,061(7.5 \%)$ & 0.11 \\
\hline Rostered & $32,192(89.1 \%)$ & $19,052(89.2 \%)$ & $13,228(86.9 \%)$ & $22,998(83.6 \%)$ & 0.16 \\
\hline Virtually rostered & $2,230(6.2 \%)$ & $1,560(7.3 \%)$ & $1,237(8.1 \%)$ & $2,461(8.9 \%)$ & 0.11 \\
\hline \multicolumn{6}{|l|}{ Physicians seen in year prior to index date } \\
\hline PCP & 31,059 (85.9\%) & $18,732(87.7 \%)$ & $13,053(85.7 \%)$ & 23,645 (85.9\%) & 0.00 \\
\hline $\begin{array}{l}\text { Psychiatrist or } \\
\text { geriatric } \\
\text { psychiatrist }\end{array}$ & $4,013(11.1 \%)$ & $2,055(9.6 \%)$ & $1,444(9.5 \%)$ & $2,617(9.5 \%)$ & 0.05 \\
\hline Geriatrician & $7,833(21.7 \%)$ & $4,599(21.5 \%)$ & 3,393 (22.3\%) & $6,090(22.1 \%)$ & 0.01 \\
\hline Neurologist & $5,205(14.4 \%)$ & $2,876(13.5 \%)$ & $2,122(13.9 \%)$ & $3,790(13.8 \%)$ & 0.02 \\
\hline $\begin{array}{l}\text { Past-Year Hospitalizations or Emergency } \\
\text { Department Visits }\end{array}$ & 16,655 (46.1\%) & $9,567(44.8 \%)$ & $6,920(45.5 \%)$ & $12,377(45.0 \%)$ & 0.02 \\
\hline \multicolumn{6}{|l|}{ Doctor-Patient Relationship Variables ${ }^{\mathrm{b}}$} \\
\hline - Annual number of visits to PCP & $8.24 \pm 8.60$ & $10.02 \pm 9.96$ & $10.84 \pm 11.10$ & $13.50 \pm 15.03$ & 0.43 \\
\hline $\begin{array}{l}\text { - High annual PCP } \\
\text { visit frequency }\end{array}$ & $6,181(17.1 \%)$ & $5,109(23.9 \%)$ & $4,183(27.5 \%)$ & $9,847(35.8 \%)$ & 0.21 \\
\hline - Continuity of care ${ }^{d}$ & $0.73 \pm 0.33$ & $0.78 \pm 0.31$ & $0.77 \pm 0.32$ & $0.76 \pm 0.33$ & 0.11 \\
\hline $\begin{array}{l}\cdot \text { High }(\geq 0.75) \\
\text { continuity of care }\end{array}$ & 22,755 (62.9\%) & $15,069(70.5 \%)$ & $10,570(69.4 \%)$ & $19,052(69.2 \%)$ & 0.13 \\
\hline - Relationship duration ${ }^{e}$ & $8.20 \pm 7.15$ & $9.86 \pm 7.42$ & $10.00 \pm 7.61$ & $9.31 \pm 7.38$ & 0.15 \\
\hline $\begin{array}{l}\cdot \text { Same } P C P \text { for } \geq 5 \\
\text { years }\end{array}$ & 20,991 (58.1\%) & $14,128(66.1 \%)$ & $10,047(66.0 \%)$ & $17,603(64.0 \%)$ & 0.12 \\
\hline \multicolumn{6}{|c|}{ a Standardised difference of means comparing people whose PCP sees $<20$ patients daily to people whose $\mathrm{PCP}$ sees $\geq 30$ patients daily } \\
\hline \multicolumn{6}{|c|}{ b Measured in two years prior to index date, from April 1, 2014 to March 31, 2016} \\
\hline \multicolumn{6}{|c|}{ c Patient made average of $\geq 7$ visits/year between April 1, 2014 to March 31, 2016} \\
\hline \multicolumn{6}{|c|}{ d Proportion of all physician visits from April 1, 2014 to March 31, 2016 that were to PCP } \\
\hline " Length of time in years patient cared for by & ent PCP & & & & \\
\hline
\end{tabular}

dementia were more likely to receive both potentially harmful (benzodiazepine and antipsychotic) and potentially beneficial (cholinesterase inhibitor) prescriptions from their PCP if that physician saw $\geq 30$ versus $<20$ patients per day. Factors that increased face-time between doctors and their patients with dementia (i.e., more frequent visits, high continuity of care) were also associated with significantly increased likelihood of both desirable and undesirable prescribing, independent of daily patient volume. In contrast, patients of high-volume physicians were less likely to receive preventive care in the form of influenza vaccinations. Patients of high-volume physicians benefited more significantly than those of low-volume physicians from frequent office visits and high continuity of care when it came to cholinesterase inhibitor prescribing and vaccinations, but not benzodiazepine and antipsychotic prescribing.

Our "high volume" cut-off of 30 is lower than "high volume" definitions used in other studies ( $>34$ to $\geq 75$ patients per day $[6,13,14]$, as well as the daily patient caps (up to 65 patients per day) [7] that have been implemented in other jurisdictions. Even so, our findings are aligned with extant evidence that shows reduced preventive care in persons with diabetes by high-volume primary care providers, $[6,15,16]$ as well as a correlation between visit frequency, continuity of care and likelihood 
Table 2 Characteristics of Primary Care Physicians (PCPS) Caring for Older Ontarians with Dementia

\begin{tabular}{|c|c|c|c|c|c|}
\hline \multirow[b]{3}{*}{ Physician Characteristics } & \multicolumn{4}{|c|}{ Average Number of Patients Seen Daily by Primary Care Physicians } & \multirow{2}{*}{$\begin{array}{l}\text { Standardized } \\
\text { Difference }(<20 \\
\text { vs } \geq 30)\end{array}$} \\
\hline & \multirow{2}{*}{$\begin{array}{l}<20 \\
N=3,884 \\
(\%)\end{array}$} & \multirow{2}{*}{$\begin{array}{l}20-24 \\
N=1,662 \\
(\%)\end{array}$} & \multirow{2}{*}{$\begin{array}{l}25-29 \\
N=1,059 \\
(\%)\end{array}$} & \multirow{2}{*}{$\begin{array}{l}\geq 30 \\
N=1,763 \\
(\%)\end{array}$} & \\
\hline & & & & & \\
\hline \multicolumn{6}{|l|}{ Panel Size } \\
\hline Mean $\pm S D$ & $988 \pm 491$ & $1,425 \pm 562$ & $1,559 \pm 646$ & $2,035 \pm 986$ & 1.34 \\
\hline Median (IQR) & $977(688-1,299)$ & $1,452(1,094-1,805)$ & $1,598(1,118-2,018)$ & $2,002(1,385-2,597)$ & \\
\hline \multicolumn{6}{|l|}{ Physician age } \\
\hline Mean \pm SD & $49.9 \pm 12.9$ & $53.2 \pm 11.9$ & $54.0 \pm 12.0$ & $54.3 \pm 11.0$ & 0.36 \\
\hline Median (IQR) & $50(39-60)$ & $54(45-62)$ & $55(45-64)$ & $54(46-62)$ & \\
\hline \multicolumn{6}{|l|}{ Physician sex } \\
\hline Female & $2,259(58.2 \%)$ & $691(41.6 \%)$ & $355(33.5 \%)$ & $469(26.6 \%)$ & 0.67 \\
\hline Male & $1,619(41.7 \%)$ & $967(58.2 \%)$ & $702(66.3 \%)$ & 1,291 (73.2\%) & 0.67 \\
\hline Missing data & $6(0.2 \%)$ & $<=5(0.2 \%)$ & $<=5(0.2 \%)$ & $<=5(0.2 \%)$ & $N / A^{a}$ \\
\hline \multicolumn{6}{|c|}{ Years since medical school graduation } \\
\hline$\leq 15$ & $1,358(35.0 \%)$ & $353(21.2 \%)$ & $208(19.6 \%)$ & $250(14.2 \%)$ & 0.50 \\
\hline $16-25$ & $854(22.0 \%)$ & $387(23.3 \%)$ & $243(22.9 \%)$ & $494(28.0 \%)$ & 0.14 \\
\hline $26-35$ & $966(24.9 \%)$ & $511(30.7 \%)$ & $281(26.5 \%)$ & $531(30.1 \%)$ & 0.12 \\
\hline$\geq 36$ & $699(18.0 \%)$ & 407 (24.5\%) & $325(30.7 \%)$ & $485(27.5 \%)$ & 0.23 \\
\hline Missing data & $7(0.2 \%)$ & $<=5(0.2 \%)$ & $<=5(0.2 \%)$ & $<=5(0.2 \%)$ & $N / A^{a}$ \\
\hline \multicolumn{6}{|l|}{ Canadian medical graduate } \\
\hline Yes & $2,913(75.0 \%)$ & $1,103(66.4 \%)$ & $619(58.5 \%)$ & $842(47.8 \%)$ & 0.58 \\
\hline No & $693(17.8 \%)$ & 465 (28.0\%) & 394 (37.2\%) & 864 (49.0\%) & 0.70 \\
\hline Missing data & $278(7.2 \%)$ & $94(5.7 \%)$ & $46(4.3 \%)$ & $57(3.2 \%)$ & 0.18 \\
\hline \multicolumn{6}{|l|}{ Practice location } \\
\hline Urban & $2,662(68.5 \%)$ & 1,203 (72.4\%) & 815 (77.0\%) & 1,463 (83.0\%) & 0.34 \\
\hline Non-urban & $600(15.4 \%)$ & $291(17.5 \%)$ & 168 (15.9\%) & $204(11.6 \%)$ & 0.11 \\
\hline Rural & $295(7.6 \%)$ & $72(4.3 \%)$ & $24(2.3 \%)$ & $35(2.0 \%)$ & 0.26 \\
\hline Missing data & $327(8.4 \%)$ & $96(5.8 \%)$ & $52(4.9 \%)$ & $61(3.5 \%)$ & 0.21 \\
\hline \multicolumn{6}{|l|}{ Payment model } \\
\hline Fee-for-service $(F F S)^{b}$ & $403(10.4 \%)$ & $132(7.9 \%)$ & 97 (9.2\%) & $255(14.5 \%)$ & 0.12 \\
\hline \multicolumn{6}{|l|}{ Enhanced FFS } \\
\hline CCM & $87(2.2 \%)$ & $55(3.3 \%)$ & $57(5.4 \%)$ & $127(7.2 \%)$ & 0.24 \\
\hline $\mathrm{FHG}$ & $504(13.0 \%)$ & 447 (26.9\%) & 391 (36.9\%) & $945(53.6 \%)$ & 0.96 \\
\hline \multicolumn{6}{|l|}{ Capitation $^{d}$} \\
\hline FHT & 1,589 (40.9\%) & 412 (24.8\%) & 164 (15.5\%) & $109(6.2 \%)$ & 0.90 \\
\hline $\mathrm{FHN}$ or $\mathrm{FHO}$ & 1,221 (31.4\%) & 609 (36.6\%) & 349 (33.0\%) & $327(18.5 \%)$ & 0.30 \\
\hline Other & $80(2.1 \%)$ & $7(0.4 \%)$ & $<=5(0.1 \%)$ & $0(0.0 \%)$ & 0.21 \\
\hline $\begin{array}{l}\text { Works Fulltime } \\
\text { (FTE } \geq 1.00)\end{array}$ & $1,665(42.9 \%)$ & 1,434 (86.3\%) & 984 (92.9\%) & 1,692 (96.0\%) & 1.41 \\
\hline
\end{tabular}

a N/A: Could not report SDs comparing cell sizes that are too small to report while maintaining confidentiality

b Fee-for-service: Income through fee for service billings. How PCPs not in payment enrollment or salary model

c Enhanced fee-for-service: Majority of income through fee for service billings with incentives for chronic disease management and preventive care. Includes: CCM: Comprehensive Care Mode, FHG: Family Health Group

d Capitation and blended capitation models: Majority of income through capitation fees. Capitation based on a defined basket of primary care services provided to enrolled patients based on age/sex of each patient. Fee-for-service paid for other services. Additionally, physicians receive monthly comprehensive care capitation payments for all enrolled patients. Includes: FHT: Family Health Team, FHN: Family Health Network, FHO: Family Health Organization

of receiving preventive testing and medicines in persons with diabetes [37, 42] and heart disease. [43, 44] Similar to our findings, Cadieux et al. showed that high-volume PCPs were more likely than low-volume PCPs to inappropriately prescribe non-indicated antibiotics, [13] and Kroll et al. showed that frequent visitors in primary care were more likely to receive benzodiazepine prescriptions [45]. 
Table 3 Adjusted Relative Rates of Vaccination and Prescriptions for People with Dementia, by PCP's daily patient volume

\begin{tabular}{|c|c|c|c|c|c|c|c|c|}
\hline \multirow[t]{2}{*}{ Outcome } & \multicolumn{2}{|c|}{ Influenza Vaccination } & \multicolumn{2}{|c|}{$\begin{array}{l}\text { Cholinesterase Inhibitor } \\
\text { Prescription }\end{array}$} & \multicolumn{2}{|c|}{ Benzodiazepine Prescription } & \multicolumn{2}{|c|}{ Antipsychotic Prescription } \\
\hline & $\begin{array}{l}\text { Adjusted }^{\mathrm{a}} \mathrm{RR} \\
(95 \% \mathrm{Cl})\end{array}$ & $p$-value & $\begin{array}{l}\text { Adjusted }^{\mathrm{a}} \mathrm{RR} \\
(95 \% \mathrm{Cl})\end{array}$ & $p$-value & $\begin{array}{l}\text { Adjusted }^{\text {a }} \text { RR } \\
(95 \% \mathrm{Cl})\end{array}$ & $p$-value & $\begin{array}{l}\text { Adjusted }^{\mathrm{a}} \mathrm{RR} \\
(95 \% \mathrm{Cl})\end{array}$ & $p$-value \\
\hline \multicolumn{9}{|c|}{ Physician's Daily Patient Volume } \\
\hline$<20$ patients/day & 1.0 (reference) & - & 1.0 (reference) & - & 1.0 (reference) & - & 1.0 (reference) & - \\
\hline $20-24$ patients/day & $\begin{array}{l}1.00 \\
\text { (0.98 to } 1.03)\end{array}$ & 0.81 & $\begin{array}{l}1.10 \\
(1.05 \text { to } 1.14)\end{array}$ & $<.0001$ & $\begin{array}{l}1.17 \\
(1.10 \text { to } 1.24)\end{array}$ & $<.0001$ & $\begin{array}{l}1.12 \\
(1.05 \text { to } 1.19)\end{array}$ & 0.001 \\
\hline 25-29 patients/day & $\begin{array}{l}0.98 \\
(0.95 \text { to } 1.02)\end{array}$ & 0.32 & $\begin{array}{l}1.09 \\
(1.04 \text { to } 1.14)\end{array}$ & 0.0002 & $\begin{array}{l}1.17 \\
(1.09 \text { to } 1.26)\end{array}$ & $<.0001$ & $\begin{array}{l}1.15 \\
(1.06 \text { to } 1.23)\end{array}$ & 0.0003 \\
\hline$\geq 30$ patients/day & $\begin{array}{l}0.97 \\
\text { (0.94 to } 0.996)\end{array}$ & 0.02 & $\begin{array}{l}1.08 \\
(1.04 \text { to } 1.13)\end{array}$ & 0.0001 & $\begin{array}{l}1.32 \\
(1.23 \text { to } 1.41)\end{array}$ & $<.0001$ & $\begin{array}{l}1.25 \\
(1.17 \text { to } 1.33)\end{array}$ & $<.0001$ \\
\hline
\end{tabular}

a All models were adjusted for the following confounders: patient age, sex, years since dementia diagnosis, CIHI Population Grouper category, urban/rural location of residence, neighborhood deprivation quintile, past 10-year immigration, and PCP age, sex, years since graduation, Canadian medical graduate, urban/rural location of practice. Models of influenza vaccination were also adjusted for past-year hospitalization for COPD

Although some high-volume PCPs work longer hours to see more patients per day, at least some of their high volume comes at the cost of visit-time per patient [10]. Benzodiazepines and antipsychotics are often prescribed to treat symptoms that are highly disruptive to people with dementia and their caregivers: sleep disturbance and other neuropsychiatric symptoms of dementia. In the face of such symptoms, the decision to withhold or discontinue treatment may require extensive discussion with patients and caregivers that high-volume physicians do not have time for [46]. Increased visit frequency and continuity of care may allow increased opportunity for primary care physicians to provide preventive care (i.e., cholinesterase inhibitors and vaccinations), but may also increase the number of times physicians are faced with caregivers and patients requesting medications to cope with distressing symptoms [47].

Our study has some limitations. We did not have data on number of hours worked by physicians; therefore, we are unable to directly assess whether shorter visit length was driving differences in outcomes between high- versus low-volume PCPs. There is, however, significant existing evidence that high-volume PCPs achieve their high volume through briefer appointments [9-11]. Our use of health administrative data limited the scope of primary care quality indicators for people with dementia we could assess; the relation of high-volume care and quality indicators such as patient or caregiver satisfaction, frequent reassessment of cognitive status and provision of caregiver support $[20,48,49]$ should be studied in subsequent work. Also, our measure of influenza vaccination has only moderate sensitivity and specificity. [35] We were only able to describe dementia type among $35 \%$ of our sample and have no data on specific neuropsychiatric symptoms and their severity for each patient. The balance of risks and benefits associated with prescription of medications to patients with dementia is determined in part by these clinical features; [50] therefore, there is some inherent misclassification of these processes when identifying them as either desirable or undesirable at a population level. Finally, patients of high-volume physicians in our study had higher rates of benzodiazepine and

Table 4 Adjusted Main Effects of Doctor-Patient Relationship variables on Vaccination and Medication Receipt in Older Ontarians with Dementia

\begin{tabular}{|c|c|c|c|c|c|c|c|c|}
\hline \multirow[t]{2}{*}{ Effects } & \multicolumn{2}{|l|}{ Influenza Vaccination } & \multicolumn{2}{|c|}{$\begin{array}{l}\text { Cholinesterase Inhibitor } \\
\text { Prescription }\end{array}$} & \multicolumn{2}{|c|}{ Benzodiazepine Prescription } & \multicolumn{2}{|c|}{ Antipsychotic Prescription } \\
\hline & $\begin{array}{l}\text { Adjusted RR }{ }^{a}(95 \% \\
\text { Cl) }\end{array}$ & $p$-value & $\begin{array}{l}\text { Adjusted RR (95\% } \\
\text { Cl) }\end{array}$ & $p$-value & $\begin{array}{l}\text { Adjusted RR (95\% } \\
\text { Cl) }\end{array}$ & $p$-value & $\begin{array}{l}\text { Adjusted RR (95\% } \\
\text { Cl) }\end{array}$ & $p$-value \\
\hline $\begin{array}{l}\text { High visit frequency } \\
\text { (>7 visits/year) }\end{array}$ & $1.32(1.30$ to 1.34$)$ & $<.0001$ & 1.09 (1.07 to 1.12$)$ & $<.0001$ & 1.74 (1.67 to 1.82$)$ & $<.0001$ & 1.25 (1.19 to 1.30$)$ & $<.0001$ \\
\hline $\begin{array}{l}\text { High continuity of } \\
\text { care }\end{array}$ & $1.19(1.16$ to 1.21$)$ & $<.0001$ & 1.47 (1.43 to 1.52$)$ & $<.0001$ & $1.42(1.35$ to 1.49$)$ & $<.0001$ & 1.31 (1.24 to 1.37$)$ & $<.0001$ \\
\hline $\begin{array}{l}\text { Long ( } \geq 5 \text { years) } \\
\text { doctor-patient } \\
\text { relationship }\end{array}$ & 1.14 (1.11 to 1.17$)$ & $<.0001$ & $1.13(1.10$ to 1.16$)$ & $<.0001$ & 1.04 (0.996 to 1.10) & 0.08 & 0.89 (0.85 to 0.94$)$ & $<.0001$ \\
\hline
\end{tabular}

Notes: ${ }^{a}$ All models were adjusted for the following confounders: patient age, sex, years since dementia diagnosis, CIHI Population Grouper category, urban/rural location of residence, neighborhood deprivation quintile, past 10-year immigration, and PCP daily patient volume, age, sex, years since graduation, Canadian medical graduate, urban/rural location of practice. Models of influenza vaccination were also adjusted for past-year hospitalization for COPD 
antipsychotic prescribing in the year prior to the study start. Although differences in baseline prescribing could explain ongoing differences in prescribing, we argue that PCPs are responsible for both initiating beneficial medications and deprescribing inappropriate medications in their patients. Given the absence of differences in all available measures of morbidity across daily patient volume groups (type/duration of dementia diagnosis, past-year hospitalizations and emergency department visits, CIHI Population Grouper comorbidity index), persistence of these prescribing differences may reflect ineffective deprescribing of potentially inappropriate medications among high-volume physicians.

A strength of our study is its inclusion of all older adults with dementia and the PCPs who care for them in Ontario's single-payer health care system. We also defined daily patient volume a priori, based on cut-points that have been proposed as thresholds of safe care.[8] Our examination of four process outcomes provides a balanced perspective on how high daily patient volume affects quality of care and our sensitivity analyses illustrate that our results are robust.

We found that high-volume primary care physicians take care of much larger panels of more deprived patients in urban settings than low-volume physicians. Although daily patient volume caps may improve some aspects of care for persons with dementia, they may not be feasible in rural districts where high volume is necessary to afford timely access to care. Development and evaluation of tools and practice models that facilitate optimal care for complex patients during rushed office visits will therefore be critical in the coming years. Similarly, models of care that reduce the significant burden of non-clinical work on physicians' daily schedules should also be investigated and supported so that family doctors may spend a larger fraction of their time caring for complex patients with dementia and other chronic diseases. The association of daily patient volume with other outcomes that can be assessed in administrative data - such as pneumococcus vaccination, fall-related injuries, long-term care home admission and death - should also be examined in subsequent studies to further interrogate the volume-quality relationship identified here.

\section{Conclusion}

As the global population of people with dementia grows, evidence is needed to support optimization of primary care for this complex patient population. This study shows that people with dementia are more likely to receive both potentially harmful and potentially beneficial prescriptions, and slightly less likely to receive beneficial vaccinations if their primary care physician sees many patients per day. Primary care physicians' daily patient volume should be considered by practitioners and policymakers seeking to affect change in quality of dementia care. Impact of daily patient volume restrictions on other aspects of care - such as access - requires ongoing study.

\section{Authors' information}

NEL is a resident physician in Internal Medicine at the University of British Columbia, and a post-doctoral fellow in Primary Care and Health Systems at ICES Toronto. VL is an analyst at ICES Toronto. RHG is Scientific Director of the Institute of Health Services and Policy Research at the Canadian Institutes of Health Research (CIHR) and a Senior Core Scientist at ICES Toronto. He is also a staff family physician at St. Michael's Hospital in Toronto and a Scientist in its Centre for Urban Health Solutions, a Professor at the University of Toronto in the Department of Family and Community Medicine, Faculty of Medicine, and Dalla Lana School of Public Health. TAS is a Canadian statistician who works as a Senior Core Scientist at ICES Toronto, as a professor in the Institute of Health Policy, Management and Evaluation of the University of Toronto, and as an adjunct professor of epidemiology and of The Dartmouth Institute for Health Policy and Clinical Practice at Dartmouth College.

\section{Abbreviations}

aRR: Adjusted relative risk; COPD: Chronic obstructive pulmonary disease; OHIP: Ontario Health Insurance Plan Database; ODB: Ontario Drug Benefit Database; PCP: Primary care physician; RECORD: Reporting of Studies Conducted Using Observational Routinely-collected Data; STROBE: Strengthening the Reporting of Observational Studies in Epidemiology.

\section{Supplementary Information}

The online version contains supplementary material available at https://doi. org/10.1186/s12875-021-01398-9.

Additional file 1. Data sources.

Additional file 2. Variable Coding.

Additional file 3. Additional Data \& analyses.

\section{Acknowledgements}

We thank Ms. Dina Skvirsky for her help preparing this manuscript for submission. Dr. Richard Glazier was supported as a Clinician Scientist in the Department of Family and Community Medicine at St. Michael's Hospital and at the University of Toronto.

\section{Authors' contributions}

All authors had full access to all of the data (including statistical reports and tables) in the study and take responsibility for the integrity of the data and the accuracy of the data analysis. NEL is the corresponding author and attests that all listed authors meet authorship criteria and that no others meeting the criteria have been omitted. NEL, TAS, and RHG conceived and designed the study. VL carried out the statistical analysis and all authors interpreted the data. NEL drafted the manuscript. All authors critically revised the manuscript 
for important intellectual content. TAS obtained funding and supervised the study. All authors read and approved the final manuscript.

\section{Funding}

This study was supported by a Canadian Institutes of Health Research (CIHR) Foundations Grant (FDN 143303), and by ICES, which is funded by an annual grant from the Ontario Ministry of Health and Long-term Care (MOHLTC). The opinions, results, and conclusions reported in this paper are those of the authors and are independent from the funding sources. No endorsement by ICES or the Ontario MOHLTC is intended or should be inferred. Parts of this material are based on data and information compiled and provided by the Canadian Institute for Health Information (CIHI). However, the analyses, conclusions, opinions, and statements expressed herein are those of the author, and not necessarily those of $\mathrm{CIHI}$. The CIHR, ICES, and the MOHLTC had no involvement in the design and conduct of the study; collection, management, analysis, and interpretation of the data; preparation, review, or approval of the manuscript; and decision to submit the manuscript for publication.

\section{Availability of data and materials}

The individual-level data underlying this study are based on records generated from the administration of Ontario's publicly funded health system. ICES has a special designation under Ontario's Personal Health Information Protection Act to use these data in studies that evaluate health care delivery and outcomes. This designation is granted by the Information and Privacy Commissioner of Ontario, and is contingent on a triennial review and ongoing oversight of the privacy practices at ICES. A variety of measures are deployed to protect the personal health information entrusted to ICES and, under the Personal Health Information Protection Act (Ontario Regulation 329/04), the underlying data are legally not allowed for public repository. The data that support the findings of this study are available from ICES but restrictions apply to the availability of these data, which were used under license for the current study, and so are not publicly available. Data are however available from the authors upon reasonable request and with permission of ICES.

\section{Ethics approval and consent to participate}

This study was performed in keeping with the Declaration of Helsinki, and was approved by the research ethics board of the Sunnybrook Health Sciences Centre that ICES is affiliated with. The requirement for all individual participants' informed consent for data use was waived by the ethics committee because ICES is a prescribed entity under Ontario law.Consent for publication.Not applicable.

\section{Competing interests}

The authors declare that they have no competing interests.

\section{Author details}

1 Department of Medicine, University of British Columbia, British Columbia, 2775 Laurel Street, 10th Floor, Vancouver V5Z 1M9, Canada. ${ }^{2}$ ICES, 2075 Bayview Ave, Toronto, ON M4N 3M5, Canada. ${ }^{3}$ Dalla Lana School of Public Health, University of Toronto, 155 College St, Toronto, ON M5T 3M7, Canada. ${ }^{4}$ Institute of Health Policy, Management and Evaluation, University of Toronto, 155 College St, Toronto, ON M5T 3M7, Canada. ${ }^{5}$ Department of Family and Community Medicine, University of Toronto, 500 University Ave, Toronto, ON M5G 1V7, Canada. ${ }^{6}$ MAP Centre for Urban Health Solutions, St. Michael's Hospital, 30 Bond Street, Toronto, ON M5B 1W8, Canada. ${ }^{7}$ Dartmouth Institute for Health Policy \& Clinical Practice, Geisel School of Medicine At Dartmouth, 74 College Street, Hanover, NH 03755, USA.

\section{Received: 14 October 2020 Accepted: 11 February 2021}

\section{Published online: 09 March 2021}

\section{References}

1. Prince M, Wimo AGM, Ali GC, Wu YT, Prina M. World Alzheimer Report 2015: the global impact of dementia: an analysis of prevalence, incidence, cost and trends. London: Alzheimer's Disease International; 2015.

2. Alzheimer's disease facts and figures. Alzheimer's \& Dementia. 2019;15(3):321-87.

3. Warrick N, Prorok JC, Seitz D. Care of community-dwelling older adults with dementia and their caregivers. CMAJ. 2018;190(26):E794-9.
4. Koller D, Hua T, Bynum JP. Treatment Patterns with Antidementia Drugs in the United States: Medicare Cohort Study. J Am Geriatr Soc. 2016:64(8):1540-8.

5. Griffith LE, Gruneir A, Fisher K, et al. Patterns of health service use in community living older adults with dementia and comorbid conditions: a population-based retrospective cohort study in Ontario, Canada. BMC Geriatrics. 2016;16(1):177.

6. Cheung A, Stukel TA, Alter DA, et al. Primary Care Physician Volume and Quality of Diabetes Care: A Population-Based Cohort Study. Ann Intern Med. 2017;166(4):240-7.

7. Medical Services Commission payment schedule. Victoria. British Columbia: British Columbia Ministry of Health; 2019.

8. BMA guidance. Controlling workload in general practice strategy: British Medical Association. 2018.

9. Zyzanski SJ, Stange KC, Langa D, Flocke SA. Trade-offs in high-volume primary care practice. J Fam Pract. 1998;46(5):397-402.

10. McDonald T, Seidel JE, Patel AB, et al. High-volume general practitioners in Alberta: a descriptive analysis. CMAJ open. 2018;6(3):E254-60.

11. Grant S, Guthrie B. Efficiency and thoroughness trade-offs in high-volume organisational routines: an ethnographic study of prescribing safety in primary care. BMJ Qual Saf. 2018;27(3):199-206.

12. Burgess DJ. Are providers more likely to contribute to healthcare disparities under high levels of cognitive load? How features of the healthcare setting may lead to biases in medical decision making. Med Decis Making. 2010;30(2):246-57.

13. Cadieux G, Tamblyn R, Dauphinee D, Libman M. Predictors of inappropriate antibiotic prescribing among primary care physicians. Can Med Assoc J. 2007;177(8):877-83.

14. Gidengil CA, Linder JA, Hunter G, Setodji C, Mehrotra A. The volume-quality relationship in antibiotic prescribing: when more isn't better. Inquiry. 2015;52:0046958015571130.

15. Bower P, Campbell S, Bojke C, Sibbald B. Team structure, team climate and the quality of care in primary care: an observational study. Quality \& safety in health care. 2003;12(4):273-9.

16. Turchin A, Shubina M, Pendergrass ML. Relationship of physician volume with process measures and outcomes in diabetes. Diabetes Care. 2007;30(6):1442-7.

17. Tarn DM, Paterniti DA, Kravitz RL, et al. How much time does it take to prescribe a new medication? Patient Educ Couns. 2008;72(2):311-9.

18. Tricco AC, Ashoor HM, Soobiah C, et al. Comparative Effectiveness and Safety of Cognitive Enhancers for Treating Alzheimer's Disease: Systematic Review and Network Metaanalysis. J Am Geriatr Soc. 2018;66(1):170-8

19. Demicheli $V$, Jefferson T, DiPietrantonj $C$, et al. Vaccines for preventing influenza in the elderly. Cochrane Database Syst Rev. 2018;2(2):CD004876.

20. Fox C, Maidment I, Moniz-Cook E, et al. Optimising primary care for people with dementia. Ment Health Fam Med. 2013;10(3):143-51.

21. American Geriatrics Society 2015. Updated Beers Criteria for Potentially Inappropriate Medication Use in Older Adults. J Am Geriatr Soc. 2015;63(11):2227-46.

22. von Elm E, Altman DG, Egger M, Pocock SJ, Gøtzsche PC, Vandenbroucke JP. The Strengthening the Reporting of Observational Studies in Epidemiology (STROBE) Statement: guidelines for reporting observational studies. Int J Surg. 2014;12(12):1495-9.

23. Benchimol El, Smeeth L, Guttmann A, et al. The REporting of studies Conducted using Observational Routinely-collected health Data (RECORD) statement. PLoS Med. 2015;12(10):e1001885.

24. Raina P, Torrance-Rynard V, Wong M, Woodward C. Agreement between self-reported and routinely collected health-care utilization data among seniors. Health Serv Res. 2002;37(3):751-74.

25. Juurlink D, Preyra C, Croxford R, et al. Canadian Institute for Health Information Discharge Abstract Database: A Validation Study. Toronto, ON: I.C.E.S; 2006.

26. Levy AR, O'Brien BJ, Sellors C, Grootendorst P, Willison D. Coding accuracy of administrative drug claims in the Ontario Drug Benefit database. Can J Clin Pharmacol. 2003;10(2):67-71.

27. Gibson D, Richards H, Chapman A. The national ambulatory care reporting system: factors that affect the quality of its emergency data. Int J Inf Qual. 2008;2:97-114.

28. Kralj B. Measuring Rurality - RIO2008_BASIC: Methodology and Results. Toronto, ON: OMA Economics Department; 2009. 
29. Glazier RH, Rayner J, A K. Examining community health centres according to geography and priority populations served, 2011/12 to 2012/13: an ICES chartbook. Toronto, ON: ICES, 2015.

30. Matheson F, van Ingen T. Ontario Marginalization Index: User guide Versuib, 12006

31. Chiu M, Lebenbaum M, Lam K, et al. Describing the linkages of the immigration, refugees and citizenship Canada permanent resident data and vital statistics death registry to Ontario's administrative health database. BMC Med Inform Decis Making. 2016;16(1):135.

32. Schultz SE, Glazier RH. Identification of physicians providing comprehensive primary care in Ontario: a retrospective analysis using linked administrative data. CMAJ open. 2017;5(4):E856-63.

33. Jakkimainen RL, Bronskill SE, Tierney MC, et al. Identification of PhysicianDiagnosed Alzheimer's Disease and Related Dementias in PopulationBased Administrative Data: A Validation Study Using Family Physicians' Electronic Medical Records. J Alzheimers Dis. 2016;54(1):337-49.

34. Stukel TA, Glazier RH, Schultz SE, et al. Multispecialty physician networks in Ontario. Open Med. 2013;7(2):e40-55.

35. Schwartz KL, Jembere N, Campitelli MA, Buchan SA, Chung H, Kwong JC. Using physician billing claims from the Ontario Health Insurance Plan to determine individual influenza vaccination status: an updated validation study. CMAJ open. 2016;4(3):E463-70.

36. Murray M, Davies M, Boushon B. Panel size: how many patients can one doctor manage? Fam Pract Manag. 2007;14(4):44-51.

37. Pentakota SR, Rajan M, Fincke BG, et al. Does diabetes care differ by type of chronic comorbidity?: An evaluation of the Piette and Kerr framework. Diabetes Care. 2012;35(6):1285-92.

38. Breslau N, Reeb KG. Continuity of care in a university-based practice. J Med Educ. 1975;50(10):965-9.

39. Waibel S, Vargas I, Coderch J, Vazquez ML. Relational continuity with primary and secondary care doctors: a qualitative study of perceptions of users of the Catalan national health system. BMC health services research. 2018;18(1):257.

40. Canadian Institutes for Health Information. Population Grouping Methodology [information sheet]. Ottawa, ON: CIHI; 2020.

41. SAS Institute Inc. The GEE Procedure. SAS/STAT ${ }^{\circledR} 143$ User's Guide. Cary, NC: SAS Institute Inc; 2017: 3104-58

42. Fenton JJ, Von Korff M, Lin EH, Ciechanowski P, Young BA. Quality of preventive care for diabetes: effects of visit frequency and competing demands. Annals of family medicine. 2006:4(1):32-9.

43. Ahmed ST, Mahtta D, Rehman H, et al. Association between frequency of primary care provider visits and evidence-based statin prescribing and statin adherence: Findings from the Veterans Affairs system. Am Heart J. 2020;221:9-18.

44. Brookhart MA, Patrick AR, Schneeweiss S, et al. Physician follow-up and provider continuity are associated with long-term medication adherence: a study of the dynamics of statin use. Arch Intern Med. 2007;167(8):847-52.

45. Kroll DS, Nieva HR, Barsky AJ, Linder JA. Benzodiazepines are Prescribed More Frequently to Patients Already at Risk for Benzodiazepine-Related Adverse Events in Primary Care. J Gen Intern Med. 2016;31(9):1027-34.

46. Tsiga E, Panagopoulou E, Sevdalis N, Montgomery A, Benos A. The influence of time pressure on adherence to guidelines in primary care: an experimental study. BMJ Open. 2013;3(4):e002700.

47. Franz CE, Barker JC, Kravitz RL, Flores Y, Krishnan S, Hinton L. Nonmedical influences on the use of cholinesterase inhibitors in dementia care. Alzheimer Dis Assoc Disord. 2007;21(3):241-8.

48. Heckman GA, Boscart VM, Franco BB, et al. Quality of Dementia Care in the Community: Identifying Key Quality Assurance Components. Canadian geriatrics journal : CGJ. 2016;19(4):164-81.

49. Perry M, Drašković I, van Achterberg T, et al. Development and Validation of Quality Indicators for Dementia Diagnosis and Management in a Primary Care Setting. J Am Geriatr Soc. 2010;58(3):557-63.

50. Yunusa I, Alsumali A, Garba AE, Regestein QR, Eguale T. Assessment of Reported Comparative Effectiveness and Safety of Atypical Antipsychotics in the Treatment of Behavioral and Psychological Symptoms of Dementia: A Network Meta-analysis. JAMA Netw Open. 2019;2(3):e190828.

\section{Publisher's Note}

Springer Nature remains neutral with regard to jurisdictional claims in published maps and institutional affiliations. 